\title{
THE BLOOD SUPPLY OF THE HUMAN SPINAL CORD
}

\author{
BY
}

\section{B. BOLTON}

From the Research Unit and Pathological Department, National Hospital, Queen Square,

$$
\text { London }
$$

(ReCEIVED 6TH MARCH, 1939)

THE problem of the blood supply of the human spinal cord was investigated in considerable detail by various workers at the end of the last century. Adamkiewicz (1881) studied particularly the course and distribution of both arteries and veins in the white and grey matter of the cord. Eight years later Kadyi (1889) repeated his work, and also studied in great detail the course and distribution of the anterior and posterior spinal arteries, their branches, and their venæ commitantes. Kadyi showed that the anterior spinal artery was formed from paired vessels, arising from the vertebral arteries, which passed caudally along the mid-line on the anterior surface of the spinal cord. Either they united almost immediately to form a single vessel or remained as paired arteries with free anastomosis as far as the mid-cervical region of the cord. This single artery then continued its course in the mid-line on the anterior surface of the cord as far as the filum terminale. He showed that the anterior spinal artery was reinforced in its course by several lateral spinal branches which passed to the cord along the anterior or posterior roots. These varied in position and number in different specimens, but in the some 50 cases which he investigated Kadyi noted that most of the lateral spinal branches found entering along the course of many of the anterior spinal roots were very small, and he presumed that they were supplying blood to the root only. However, a few of the lateral spinal vessels were larger, and appeared to carry blood to the anterior spinal artery. Such branches were most common at the level of the 5th or 6th cervical segment and in the lower thoracic or upper lumbar level. They were usually unpaired. Near the termination of the anterior spinal artery at the filum terminale, two large vessels passed laterally and communicated lateral to the posterior spinal roots with the posterior spinal arteries. Kadyi showed that the posterior spinal arteries arose either from the vertebral or from the posterior inferior cerebellar arteries. They then passed caudally and were sometimes joined by one or more lateral spinal branches which reached the cord via the posterior roots at the level of the 4 th -7 th cervical segments. There was a very free anastomosis between the two posterior arteries across the posterior 
surface of the cord. He noted the presence of one or more large posterior lateral spinal vessels in the thoracic and lumbar regions; but in most of his cases they were fewer in number on the posterior than on the anterior surface of the cord. These investigations were all carried out by injection methods, which caused complete filling of all the vessels, as the problem was studied from a purely anatomical standpoint. No attempt was made to determine the regional blood supply of the cord.

The fact that in pressure lesions of the thoracic cord the sensory symptoms often extend to a higher level than is explicable by the site of the pressure, thereby misleading the observer as to the level of the lesion, prompted a further investigation of the exact distribution and direction of the blood flow in the spinal arteries. It was also considered that a more detailed knowledge of the vascular supply might be of value in explaining the wasting of the hand and arm muscles in cases of pressure at high cervical levels. This paper records the methods and results obtained in a series of experiments undertaken to demonstrate the direction and distribution of flow in spinal blood-vessels.

\section{Methods}

The investigations were carried out on a series of spinal cords obtained at autopsy as soon as possible after death, from subjects who during life had remained free from symptoms referable to a spinal lesion. The arterial supply was studied by means of injection of various media. In the first series coloured celloidin dissolved in acetone was the injection mass; the vessels were injected with the cord in situ. In the second series, following the removal of the cord the selected vessels were injected with a solution of indian ink.

The method employed in the celloidin injection experiments was that described by Morison (1923), who used an acetone solution of celloidin to which had been added a small quantity of camphor to prevent the celloidin becoming too brittle when hardened. The solution employed in the present experiments consisted of acetone 100 c.c., celloidin $3 \mathrm{gm}$., camphor $2 \mathrm{gm}$., and a suitable dye. The dyes used were alkanin for red coloration and a mixture of brilliant green and crystal violet for blue coloration.

The apparatus for injection consisted of a small pressure bottle, containing mercury, in which the pressure recorded by a manometer could be raised to $600 \mathrm{~mm}$. $\mathrm{Hg}$. The bottle was connected by pressure tubing with three 250 -c.c. bottles, the first containing saline, while the other two contained the red or the blue injection medium. Hypodermic needles of different bores, the points being cut off and the ends rounded with solder, were used as cannulie. The needle was tied into the selected artery and connected with the bottle containing saline. The pressure was then raised to $350 \mathrm{~mm}$. $\mathrm{Hg}$. and the vessels irrigated with saline until the outflowing fluid was no longer blood-stained. The needle was then connected with one of the botrles containing the medium and the pressure raised to $450 \mathrm{~mm}$. $\mathrm{Hg}$. These bottles were so connected with the pressure system that simultaneous injection with the media of different colours could be carried out. After fifteen minutes the pressure was released and the cord removed and fixed in formol saline for a week.

In the second series the cord, in some cases together with the midbrain and cerebellum, was removed before injection. A fine hypodermic needle was tied into the selected vessel and the local vascular system washed out with distilled water from a hypodermic syringe. When the outflowing fluid was clear a solution of water-soluble indian ink diluted to one in four with 5 per cent. formol saline and which had been twice filtered was injected. By applying the pressure manually the rate of inflow of dye medium could be easily regulated and the direction observed. 


\section{Results}

Series I.-In two cases the aorta was opened and the cannula tied into the 9 th right intercostal artery. The vessel was clamped with a silver clip just distal to the origin of the spinal branch; all the other intercostal arteries were left patent. The system was then irrigated with saline until the emerging fluid was no longer blood-stained. The other intercostal arteries were then clamped and the dye was injected. In both cases great difficulties were encountered in confining the injection medium to the selected artery, owing to the very free anastomosis of vessels around the vertebral column and in the vertebral muscles. Nevertheless, the injection medium was found to have entered the spinal arteries on the surface of the cord, and also on subsequent section of the cord it was found to have penetrated to some of the larger arteries in its substance. The anterior spinal artery in each case contained the medium from the site of injection down to the filum terminale. In one specimen (Fig. 1) the dye had, in addition, filled the anterior spinal artery rostrally for two segments to the level of the 7th thoracic segment. This portion of the vessel was much narrower in calibre than that passing caudally. On the posterior surface the posterior spinal arteries were found injected at the level of the 9th and 10th thoracic segment. There was no dye in the vessels at the 7th segment, but from the 7 th segment, where the injection of the anterior spinal artery ceased, to the 4th segment the dye was present in the posterior spinal arteries. No dye was found in the posterior spinal vessels below the site of the injection. These cases showed that the direction of flow of fluid in the anterior spinal artery in the region of the mid-thoracic cord tended to be in a caudal rather than in a rostral direction, whereas in the posterior spinal arteries the direction of flow appeared to be in the opposite direction.

Series II.-In three cases the vertebral artery was injected. In two of these cases the artery was exposed at the base of the neck at its origin from the subclavian artery and clamped proximal to the point of injection to obviate the possibility of the dye passing back into the systemic circulation. In the third case both vertebral arteries were injected simultaneously with contrasting dyes. In a fourth specimen the left vertebral artery was tied at the base of the skull proximal to passing through the foramen magnum. The vessel was then injected as in the previous experiments near its origin from the subclavian artery. In all cases the basilar artery was also clamped.

In the first two cases of series II the anterior spinal artery was found to be injected from its origin in the posterior fossa to the level of the 1st and 4th thoracic segments respectively, while the posterior spinal arteries were injected down to the level of the 5th and 6th cervical segments only (Fig. 2). The case where two colours were injected simultaneously showed an interesting distribution : from the right vertebral artery, which was injected with red celloidin, the dye had filled both anterior and posterior spinal arteries to the level of the 3rd cervical segment, while the blue dye from the left vertebral had filled the anterior spinal artery from the 3 rd cervical to the 2 nd thoracic segments, and on the posterior surface had entered a large posterior spinal branch at the 4th cervical 
segment, to fill the left posterior spinal artery at the levels of the 4th and 5th cervical segments only. In the specimen with the vertebral tied at the base of the skull the anterior spinal artery was found to be injected from the 3rd cervical to the 3rd thoracic segment. None of the dye had reached the posterior vessels (Fig. 3).

In this series of cases the anterior spinal artery received dye from two sources. The upper portion, from its origin from the vertebral artery to the

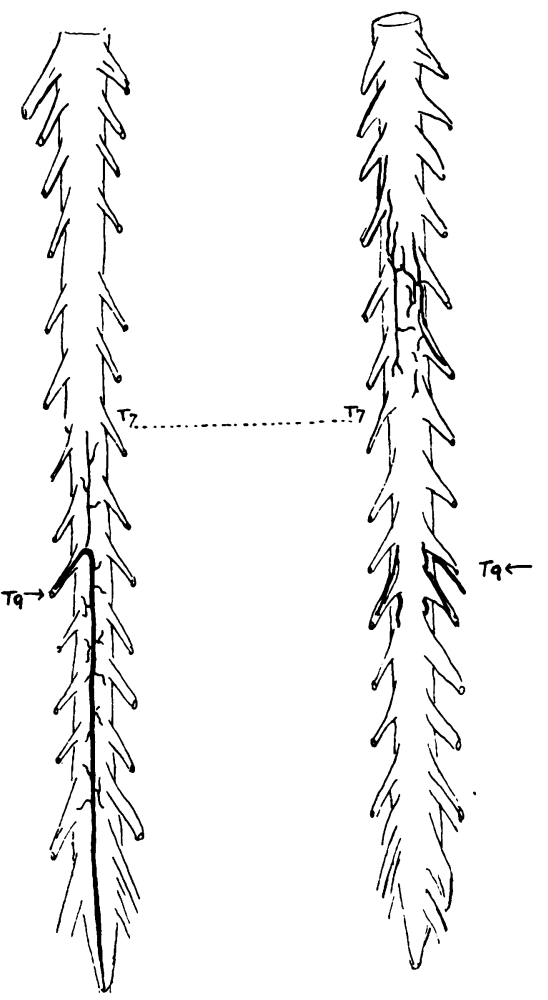

Anterior

Posterior

Fig. 1.-Drawing of cord injected in situ with celloidin solution through the 9th intercostal artery. Note that dye has passed caudally along the anterior spinal artery from site of injection to filum terminale. Rostrally it passed to $\mathrm{T} 7$ and thence to the posterior spinal artery from $\mathrm{T} 7$ to $\mathrm{T} 4$.

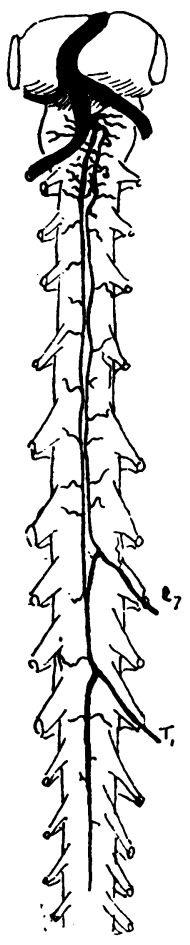

Anterior

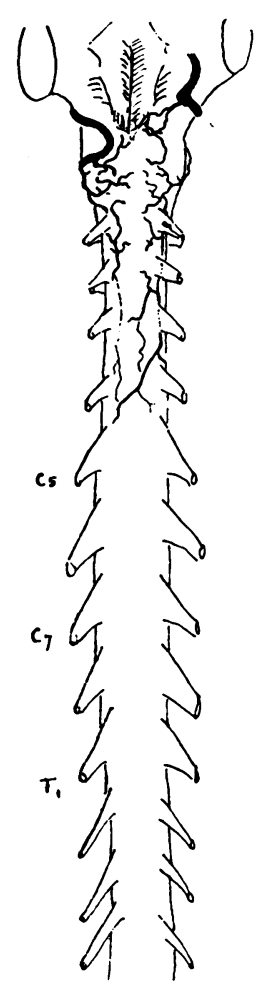

Posterior

Fig. 2.-Drawing of mid-brain and cord, injected in situ with celloidin solution through right vertebral artery at its origin from subclavian artery.

level of the 3rd cervical segment, was supplied from the one vertebral artery, while the more caudal portion, from the 3 rd cervical to the 1 st or 2 nd thoracic segments, was supplied via lateral spinal vessels originating from the other vertebral artery. This vertebral artery also supplied dye to the posterior spinal arteries and passed caudally to the lowest cervical levels.

Injection experiments were then carried out on the isolated cord by the indian ink injection method. In the first of these experiments the objective was the injection of the posterior spinal arteries, so the right vertebral artery was 
clamped between the origins of the posterior inferior cerebellar and the anterior spinal arteries. The posterior inferior cerebellar artery was clamped just before it entered the cerebellum, and the injection cannula tied into the basilar end of the vertebral artery. The results showed injection of the posterior spinal arteries to the level of the 1st thoracic segment in each case (Fig. 4). A further injection was carried out on these specimens via a lateral spinal artery at the level of the 12 th thoracic or the 1 st lumbar segments ; the anterior

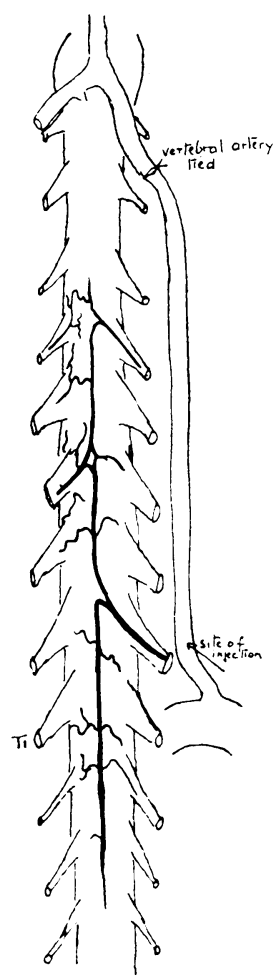

Fig. 3.-Drawing of cervical cord, injected in situ with celloidin solution through right vertebral artery. Artery clamped at base of skull. Note that anterior spinal artery is injected from C3 to T4. No vessels were injected on posterior surface.

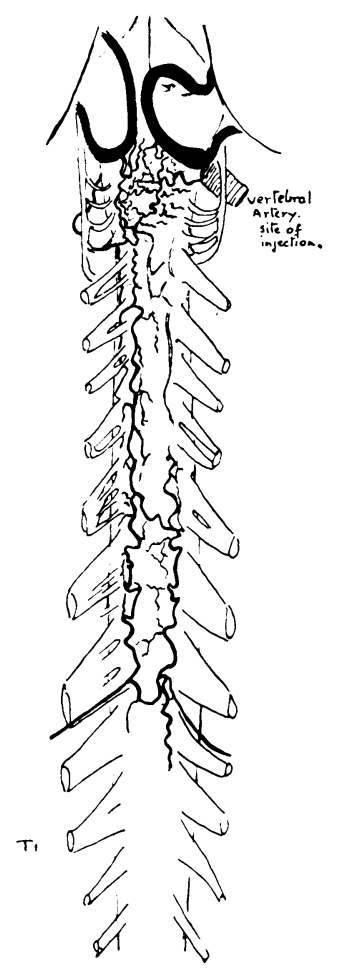

Fig. 4.-Drawing of mid-brain and cord showing result of injection of right posterior spinal artery via vertebral artery in isolated specimen.

spinal artery was clamped above the level of the second injection. During the process the dye was seen to pass down the anterior spinal artery to the point at the filum terminale where it gave rise to the two large lateral branches, which communicated with the posterior spinal arteries. The dye was then seen to fill the posterior spinal vessels from below upwards eventually to reach the level of the 4th or 3 rd thoracic segment.

In two specimens of isolated cord an anterior spinal branch at the level of the 10th thoracic segment was injected. In previous experiments it had been observed that, although the greater quantity of the injection fluid was 
observed to flow along the anterior spinal artery caudal to the site of the injection, there was often a slow filling of the anterior spinal vessel in a rostral direction. This filling was of the superficial vessel only and did not penetrate into the substance of the cord, as shown later by section of the cord. In order, however, to remove the possibility of the dye passing by a collateral circulation from this part of the artery to the posterior spinal arteries in their rostral course,

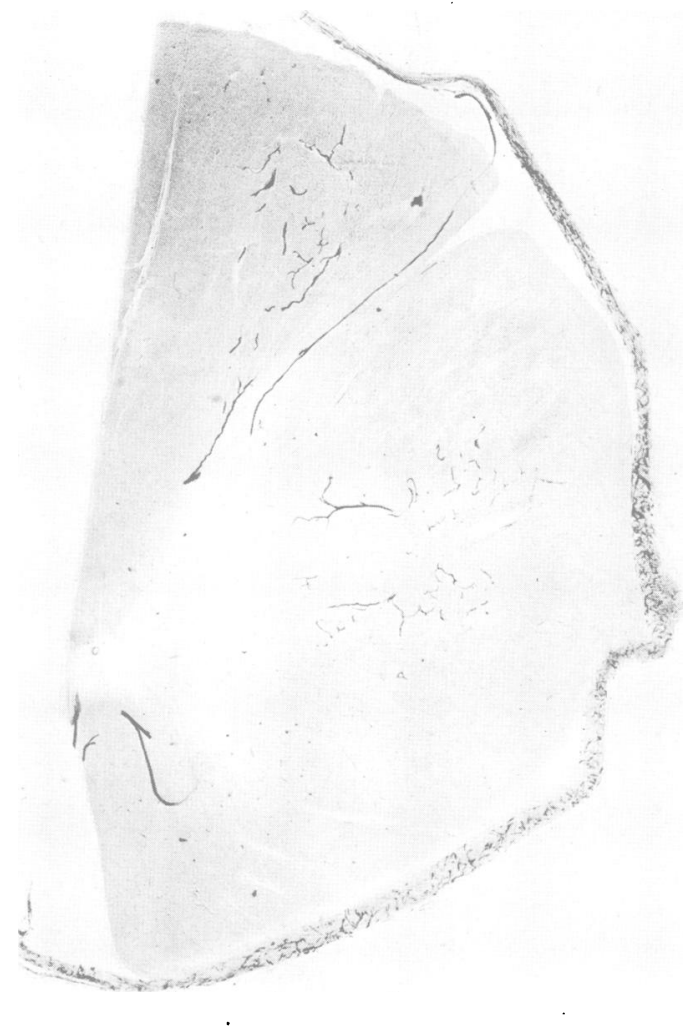

Fig. 5.-Photograph of section of cervical cord showing distribution of posterior spinal artery. Note that posterior two-thirds of posterior horn and posterior half of posterior columns only are injected.

as in Fig. 1, the anterior spinal artery was clamped above the site of injection. These findings confirmed the observations in the last experiments. The posterior spinal arteries were filled from their origin from the anterior spinal artery caudal to the 5th sacral roots, and the dye finally reached the level of the 2nd thoracic segment.

All the specimens were sectioned and examined microscopically, with the object of endeavouring to determine the areas of distribution of supply within 
the cord of the anterior and posterior spinal arteries. Fig. 5 shows a section taken from the specimen shown in Fig. 4, at the level of the 3rd cervical segment. The vessels in the posterior two-thirds of the posterior horn were injected, and also those in the posterior half of the posterior columns. Some dye had also reached the area of the pyramidal tracts, probably from a collateral arteriole

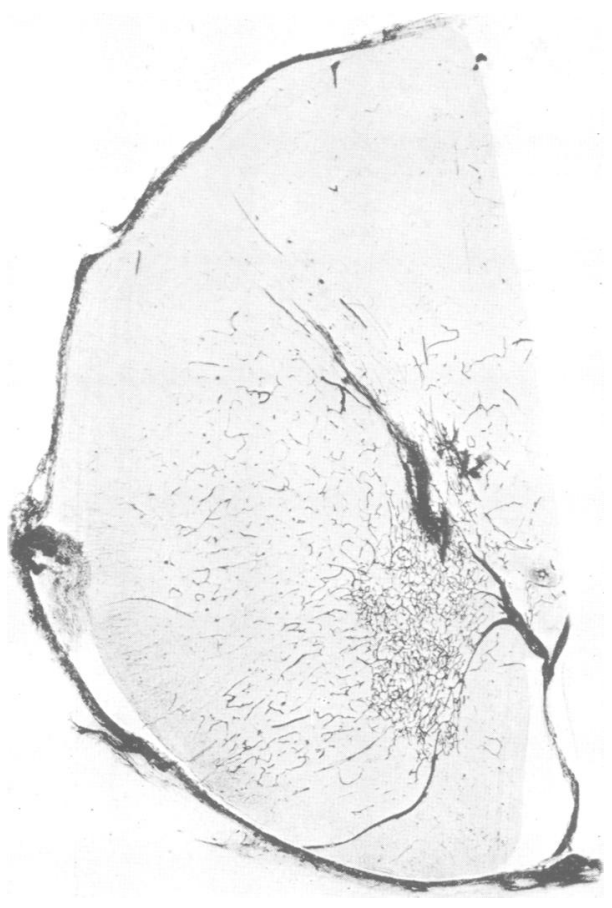

Fig. 6.-Photograph of section of cervical cord showing distribution of anterior spinal artery. Note that all the cord is injected except posterior portion of posterior horn and posterior columns.

which was passing laterally from the posterior spinal artery on that side. Fig. 6 shows a section at the same level taken from a specimen in which the anterior spinal artery only was injected. In this section the vessels in the whole of the cord, grey and white matter, are seen to be injected, except those in the posterior two-thirds of the posterior horn and the posterior half of the posterior columns. From the examination of these and similar specimens, it appeared that the anterior spinal artery supplied the greater part of the grey and white matter of the cord, while the posterior spinal artery supplied only the posterior parts of 
the posterior horn and posterior columns (Fig. 7). In order to check these results further, sections of spinal cord taken from various levels were stained by Pickworth's benzidine method, as modified by Doherty, Suh, and Alexander (1938). Fig. 7 shows a section taken from the lower cervical cord. In all the sections examined an area was observed in the posterior horn, marked $a-b$ in the photograph, where the capillaries were less numerous and the anastomosis

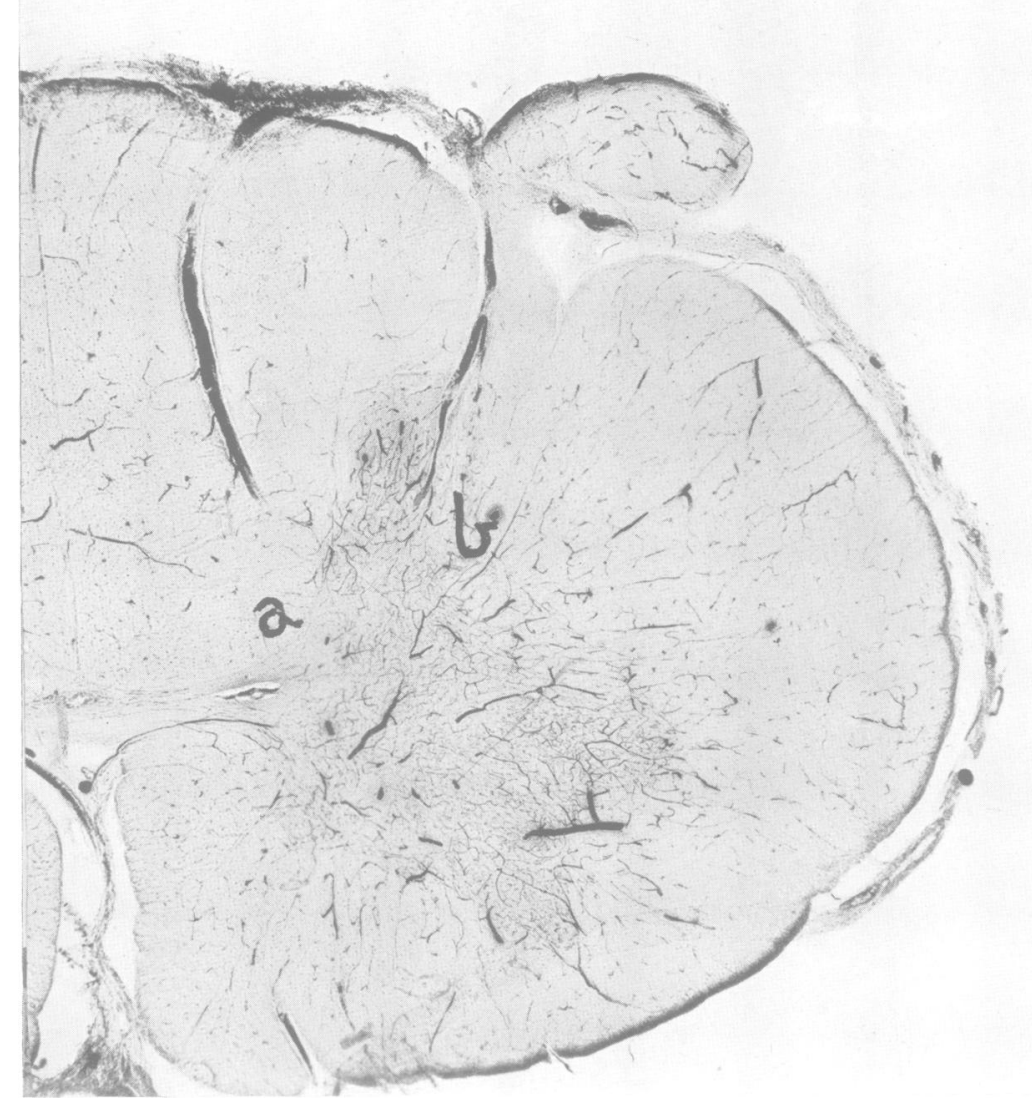

Fig. 7.-Photograph of section of cervical cord stained by Pickworth's method. Note that area $a-b$, showing comparative poverty of capillary anastomosis.

less free than in the rest of the grey matter. This area corresponded in position to the limit of supply of the anterior and posterior spinal vessels respectively, as already shown by injection of indian ink. These observations seemed to confirm the determination of the respective areas of blood supply from the anterior and posterior spinal arteries within the substance of the cord, as suggested by the diagram in Fig. 8 .

\section{Discussion}

In summarizing and reviewing the results of injection of the arterial spinal cord it is clear that in every case the usual direction of blood flow in the anterior 
spinal artery was apparently from above downwards. In the upper part of the cervical cord the supply was from the intradural portion of the vertebral artery, while the part from the 3rd cervical segment to the 1 st or 2 nd thoracic segment derived its supply from the spinal branches which arose from that portion of the artery which lay in the intravertebral canal. Below that level, namely from the upper thoracic region to the filum terminale, the supply was from a varying number of spinal vessels arising from the intercostal and lumbar arteries. It was noted that the anterior spinal artery became very small just above the point where a large lateral spinal vessel joined it : at the point where the latter

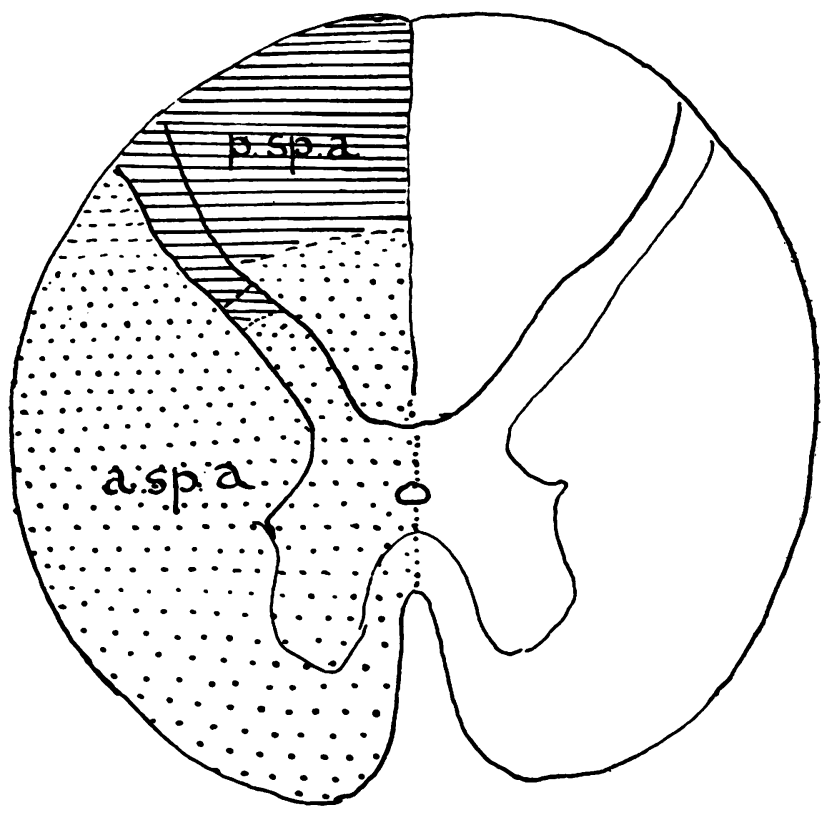

Fig. 8.-Schematic drawing of cord showing areas supplied by posterior spinal artery (lined area) and anterior spinal artery (dotted area) respectively.

joined the anterior spinal artery the main artery was of comparatively wide calibre, thus facilitating a downward rather than an upward flow of blood.

On the posterior surface, however, the vascular supply appeared to be entirely different. All the experiments showed that down to the level of the lower cervical or upper thoracic segments the flow was from above downwards, and derived in the greater part from the vertebral artery or from the posterior inferior cerebellar artery. At lower levels of the cord, however, the direction of flow seemed to be in the opposite direction. In each instance where injection of a lateral spinal artery was carried out, a filling of the posterior spinal vessels above the site of injection occurred, and in those cases where the injection was most completely carried out the main source of supply to these vessels seemed to be from the most caudal portion of the anterior spinal artery, via the two terminal branches of this vessel. These branches passed in a dorsal direction caudal to the 5th anterior sacral roots, and anastomosed 
one with each posterior spinal artery, lateral to the 5th posterior sacral roots. Although numerous small arterioles arose from the anterior spinal artery throughout the length of the cord and passed to the lateral surfaces, they appeared to make little anastomosis with the posterior spinal arteries. These arterioles were of very narrow calibre and only supplied the white matter on the lateral aspect of the cord. Large posterior lateral spinal branches were occasionally found, but normally these vessels were so small and insignificant that it was not possible to inject them directly, even with the finest hypodermic syringe. This agrees with Kadyi's observations.

The calibre of the anterior spinal artery at different levels of the cord varied

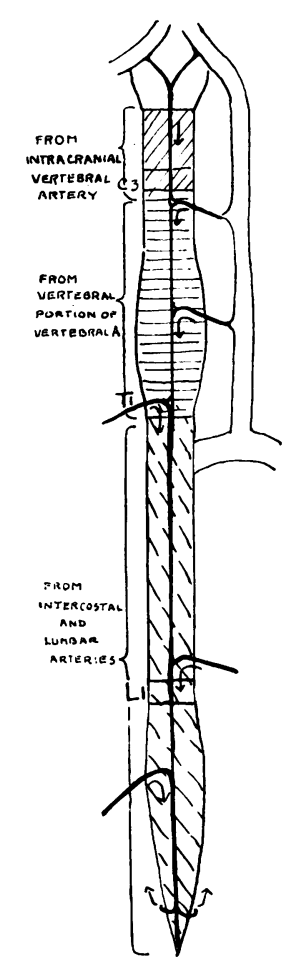

Anterior

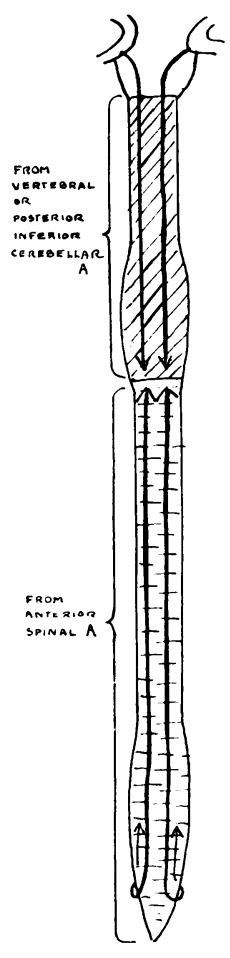

Posterior

Fig. 9.-Schematic drawing of cord showing source of supply and direction of flow of arterial blood in the spinal cord.

considerably. It appeared to be of very small dimensions immediately above the entry of a lateral spinal artery. In this way a mechanical barrier to the upward flow of blood was formed. On the posterior surface similar narrowing of the vessels was not observed, and the blood from the vertebral arteries reached the level of the highest thoracic segments in an uninterrupted flow. At this point the vertebral flow appeared to cease, and the blood below this level was derived from the caudal part of the anterior spinal artery. This ascending flow of blood was occasionally reinforced by one or more posterior lateral spinal branches entering along one of the lumbar roots. The arteries supplying the posterior surface of the lower cervical or upper thoracic segments thus 
appeared to be in the nature of end-arteries (Fig. 9). Consequently the tissues at this level, where the flow of blood from the two sources met, were likely to suffer from occlusion of one or other source. On the anterior surface, similar zones occurred just above the entry of a lateral spinal artery, the most marked of which was to be found at the lower limit of the vertebral supply, namely, at the level of the upper thoracic segments.

It might therefore be expected that in any lesion which caused pressure on the course of these vessels the first parts of the cord to suffer from the resulting ischæmia would be, not the segments at the site of pressure, but those which were situated farthest from the source of blood flow. This theory affords an explanation of the wasting which occurs in the hands and the pains and tingling found in association with pressure at the upper cervical region of the spinal cord.

The region of supply of the posterior spinal arteries is limited to the posterior portions of the posterior columns and the posterior horns, while the anterior spinal artery supplies the rest of the cord, both grey and white matter. There appears to be little or no anastomosis between the capillaries of the two arterial systems. Since the anterior spinal artery provides the main source of blood supply to the posterior spinal arteries below the upper thoracic level, partial occlusion of this vessel may cause not only an interference with the blood supply to the anterior surface of the cord below the level of the lesion, but also an ischæmia of the posterior surface of the cord to a level above that of the site of the lesion. The result would be an alteration or loss of sensation at levels higher than that of the actual site of the pressure lesion.

\section{Summary}

In a series of specimens of human spinal cord taken from subjects free from spinal lesions, the source of vascular supply and direction of blood flow of both anterior and posterior aspects of the spinal cord has been studied.

The anterior spinal artery derives its blood from the vertebral arteries and from branches of the intercostal arteries, the direction of flow being from above downwards. The blood supply to the posterior spinal arteries down to the lower cervical level arises from vertebral or posterior inferior cerebellar arteries, and for the rest of their course from the terminal portion of the anterior spinal artery, via its lowest lateral branches, the flow being directed upwards as far as the upper thoracic level. The relationship of these observations to the findings in pressure lesions of the cord has been discussed.

The areas of distribution of the anterior and posterior spinal arteries within the white and grey matter of the cord have been investigated. The posterior spinal arterial supply is limited to the posterior portion of the posterior columns and the posterior horn, while the anterior spinal artery supplies the rest of the cord substance. 
The author's thanks are due to Dr. J. G. Greenfield for his advice and kind assistance in providing material and to Dr. E. A. Carmichael for his helpful guidance and criticism.

\section{REFERENCES}

Adamkiewicz, A. (1881). Tr. int. med. Congr., 7th Sess., London, 1, 155. Doherty, M. M. Suh, T. H., and Alexander, L. (1938). Arch. Neurol. Psychiat., Chicago,

Kadyi, H. (1889). Über die Blutgefässe des menschlichen Rückenmarkes. Gubrynowicz and Schmidt, Lemberg.

Morison, D. M. (1923). J. Amer. med. Ass., 81, 178. 